\title{
Muon collider interaction region design
}

\author{
Y. I. Alexahin, E. Gianfelice-Wendt, V. V. Kashikhin, N. V. Mokhov, and A. V. Zlobin \\ FNAL, Batavia, Illinois 60510, USA
}

V. Y. Alexakhin

JINR, Dubna 141980, Russia

(Received 28 October 2010; published 2 June 2011)

\begin{abstract}
Design of a muon collider interaction region (IR) presents a number of challenges arising from low $\beta^{*}<1 \mathrm{~cm}$, correspondingly large beta-function values and beam sizes at IR magnets, as well as the necessity to protect superconducting magnets and collider detectors from muon decay products. As a consequence, the designs of the IR optics, magnets and machine-detector interface are strongly interlaced and iterative. A consistent solution for the $1.5 \mathrm{TeV}$ center-of-mass muon collider IR is presented. It can provide an average luminosity of $10^{34} \mathrm{~cm}^{-2} \mathrm{~s}^{-1}$ with an adequate protection of magnet and detector components.
\end{abstract}

DOI: 10.1103/PhysRevSTAB.14.061001

PACS numbers: 29.20.db, 84.71.Ba

\section{INTRODUCTION}

A muon collider (MC)—proposed by Budker and Skrinsky more than 40 years ago [1] — has been extensively studied in the U.S. during the past two decades [2,3]. It is now considered the most exciting option for the energy frontier machine in the post-LHC era. It has a number of important advantages over its competitor $e^{+} e^{-}$collider: potentially higher energy, better energy resolution, larger cross section for scalar particle production, smaller footprint, etc. [4]. However, in order to achieve a competitive level of luminosity, a number of demanding requirements to the collider optics and the interaction region (IR) hardware should be satisfied arising from short muon lifetime and from relatively large values of the transverse emittance and momentum spread in muon beams that can realistically be obtained with ionization cooling [3].

Challenging as they are, these requirements are aggravated by limitations on the magnet maximum operating fields as well as by the necessity to protect superconducting magnets and collider detectors from muon decay products [5]. Therefore a holistic approach to the IR design should be developed tying together optics, magnet, and shielding considerations.

The result of such an approach to the IR design of a muon collider with $1.5 \mathrm{TeV}$ center-of-mass energy and an average luminosity of $10^{34} \mathrm{~cm}^{-2} \mathrm{~s}^{-1}$ is presented in this paper. The particular value of the collision energy was chosen based on expectations of new physics at $1 \mathrm{TeV}$, though the future LHC results may point to a higher energy.

Published by the American Physical Society under the terms of the Creative Commons Attribution 3.0 License. Further distribution of this work must maintain attribution to the author(s) and the published article's title, journal citation, and DOI.

\section{IR LATTICE}

The major problem to solve is correction of the IR quadrupoles chromaticity in such a way that the dynamic aperture remained sufficiently large and did not suffer much from strong beam-beam effects.

To achieve these goals, a solution was proposed in the past based on special chromatic correction sections (CCS) with compensated spherical aberrations [6]. Each CCS includes two sextupoles separated by a $-I$ transformation so that their nonlinear kicks cancel out. There is an independent CCS for each transverse plane making the total of four chromaticity correction sextupoles on each side of the IP.

This approach has led to a number of muon collider designs, the best performance was demonstrated by a $4 \mathrm{TeV}$ center-of-mass collider design by Oide [7]. According to it, the vertical $\beta$ function in the final focus (FF) triplet is much larger than the horizontal one (up to $900 \mathrm{~km}$ for $\beta^{*}=3 \mathrm{~mm}$ ) and its chromatic perturbation is corrected first by a CCS starting at $180^{\circ}$ vertical phase advance from the source (FF quads). However, very large $\beta$-function values together with large overall phase advance make the optics too sensitive to magnet field errors and misalignments.

\section{A. Chromatic correction scheme}

In order to clarify the principle of the proposed scheme in this paper, let us first recall the definition of the Montague chromatic functions [8]:

$$
\begin{aligned}
& A_{z}=\frac{\partial}{\partial \delta} \alpha_{z}-\alpha_{z} B_{z}, \quad B_{z}=\frac{1}{\beta_{z}} \frac{\partial}{\partial \delta} \beta_{z}, \\
& W_{z}=\sqrt{A_{z}^{2}+B_{z}^{2}}, \quad \delta=\Delta p / p, \quad z=x, y .
\end{aligned}
$$

The form of equations which these functions obey depends on the set of dynamic variables used. With the choice of (noncanonical) pairs $\left(z, z^{\prime}\right)$ these equations are 


$$
A_{z}^{\prime}=2 \varphi_{z}^{\prime} B_{z}-\beta_{z} k, \quad B_{z}^{\prime}=-2 \varphi_{z}^{\prime} A_{z},
$$

where $\varphi_{z}$ is the betatron phase advance, $k= \pm\left(K_{1}-\right.$ $D_{x} K_{2}$ ) for $z=x, y, K_{1}$ and $K_{2}$ are normalized by $B \rho$ quadrupole and sextupole gradients, the prime denotes differentiation by path length.

Equation (2) shows that initially only the Twiss $\alpha$ functions are perturbed, but as the betatron phase advance increases this initial perturbation, if left uncompensated, will be converted into a more dangerous perturbation of $\beta$ functions. To prevent this, the correction sextupole must be placed at the same phase advance as the quadrupoles.

Figure 1 presents the IR layout which realizes this idea for the vertical plane, the horizontal chromatic function is much smaller (see Fig. 1 lower plot) and can be corrected farther from the IP. Dipoles (shown at the top as orange rectangles) are placed next to the FF quadrupoles (blue rectangles) and generate a sufficiently large dispersion function at the S1 sextupole location. To increase dispersion the quadrupoles are displaced by $\sim 1 / 10$ aperture providing up to $2 \mathrm{~T}$ bending field. The lattice is symmetric with respect to the IP so that only the right half is shown.

Another principal difference of the proposed design is that we avoid using an error-prone CCS for the vertical plane relying only on smallness of the horizontal $\beta$ function at the S1 sextupole location: both resonance driving terms and detuning coefficients produced by a normal sextupole contain powers of $\beta_{x}$ and can be reduced with its help.

Such a recipe does not work for the horizontal plane: smallness of $\beta_{y}$ at a normal sextupole location is beneficial but does not suppress horizontal aberrations, so a CCS is still necessary with $-I$ separated sextupole pair (marked as S2 and S4 in Fig. 1). Thus, there is a total of three sextupoles on each side of the IP for the Montague chromatic functions correction.
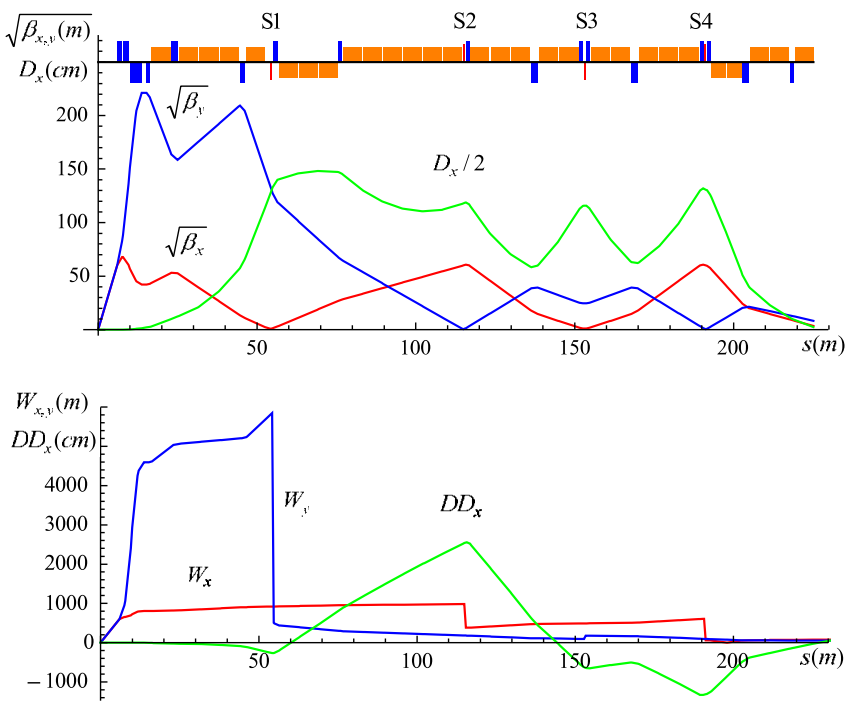

FIG. 1. IR layout and optics functions (top) and chromatic functions (bottom).
Correction of these functions which is important by itself also reduces the higher order chromaticity, i.e., the nonlinear dependence of betatron tunes on momentum. For the second order chromaticity we have [9]

$$
\chi_{z}^{(2)}=\frac{1}{8 \pi} \int_{0}^{C}\left(-k B_{z} \pm 2 K_{2} \frac{d D_{x}}{d \delta}\right) \beta_{z} d s-\chi_{z}^{(1)}
$$

with $\chi_{z}^{(1)}$ being the linear chromaticity, $z=x, y$.

Equation (3) shows that the second order dispersion, $d D_{x} / d \delta$, also needs to be corrected. This is achieved by adjusting the relative values of the first order dispersion at sextupoles S2 and S4 and by installing an additional sextupole, S3, at the center of the horizontal CCS (Fig. 1).

This additional sextupole signifies the final departure from the concept of noninterleaved sextupole families which has also been abandoned in the design of the bending $\operatorname{arcs}[10]$.

\section{B. Lattice performance}

Basic parameters of the muon beams and the collider lattice are given in Table I. With relatively large emittances expected from the cooling channel and short bunch length, the rms energy spread reaches $0.1 \%$ so that a momentum acceptance of at least $\pm 0.3 \%$ is required.

Figure 2 shows the dependence on momentum of betatron tunes and momentum compaction factor obtained with some help from additional octupole and decapole correctors placed in the CCS. The stability range of $\pm 1.2 \%$ significantly exceeds the minimum requirement.

Problems with the dynamic aperture (DA) and beambeam effect in a muon collider are significantly alleviated by the fact that muons will be dumped after less than 2000 turns (see Sec. IV). In the result, the high order resonances have little chance to show up. Preliminary studies [10] using MAD code demonstrated a good dynamic aperture $(\sim 5 \sigma)$ in the absence of magnet imperfections and

TABLE I. Baseline muon collider parameters [10].

\begin{tabular}{lll}
\hline \hline Parameter & Unit & Value \\
\hline Beam energy & $\mathrm{TeV}$ & 0.75 \\
Repetition rate & $\mathrm{Hz}$ & 15 \\
Average luminosity/IP & $10^{34} / \mathrm{cm}^{2} / \mathrm{s}$ & 1.1 \\
Number of IPs, $N_{\mathrm{IP}}$ & & 2 \\
Circumference, $C$ & $\mathrm{~km}$ & 2.73 \\
$\beta^{*}$ & $\mathrm{~cm}$ & $1(0.5-2)$ \\
Momentum compaction, $\alpha_{p}$ & $10^{-5}$ & -1.3 \\
Normalized rms emittance, $\varepsilon_{\perp N}$ & $\pi \mathrm{mm}$ mrad & 25 \\
Momentum spread, $\sigma_{p} / p$ & $\%$ & 0.1 \\
Bunch length, $\sigma_{s}$ & $\mathrm{~cm}$ & 1 \\
Number of muons/bunch & $10^{12}$ & 2 \\
Beam-beam parameter/IP, $\xi$ & & 0.09 \\
rf voltage at $800 \mathrm{MHz}$ & $\mathrm{MV}$ & 16 \\
\hline \hline
\end{tabular}



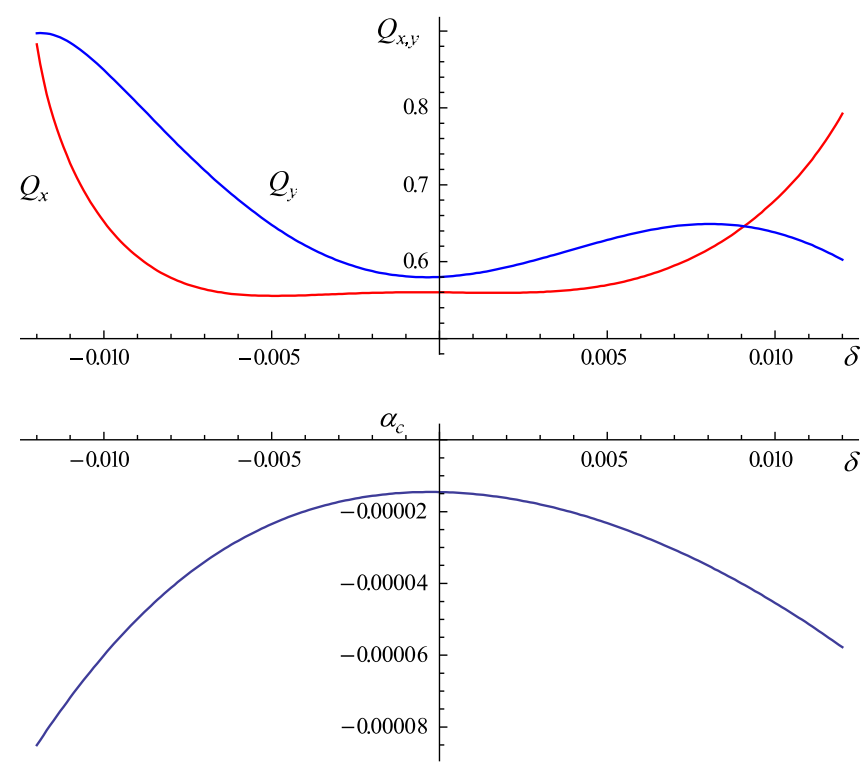

FIG. 2. Fractional betatron tunes (top) and momentum compaction factor (bottom) vs momentum.

beam-beam effect and only a modest DA reduction with the beam-beam parameter as large as 0.09 per IP. ${ }^{2}$

The presented design raises a number of questions: large values of vertical $\beta$ function and therefore of the vertical beam size in the IR quads and dipoles make it necessary to reconsider earlier magnet designs, closeness of the dipoles to IP may complicate the detector protection from $\gamma$ radiation emitted by decay electrons and positrons and from these electrons and positrons themselves.

These issues as well as problems with heat deposition in the magnet coils are considered in the subsequent sections.

\section{IR MAGNET DESIGN}

Figure 3 shows vertical and horizontal sizes of the muon beam corresponding to parameters from Table I and the inner radii of closest to IP magnets determined by the requirement $a>5 \sigma_{\max }+1 \mathrm{~cm}$. A $5 \sigma$ aperture radius may seem too small compared to $9 \sigma_{\max }$ aperture adopted for the LHC IR upgrade [11]. However, one should keep in mind that in $\mathrm{MC}$ there is no crossing angle and, due to the short time the muons spend in the collider, there will be practically no diffusion so that the beams can be collimated at less than $4 \sigma$ amplitudes; the remainder providing room for possible closed orbit excursions. In the actual magnet design, the bore radius was increased by an additional $5 \mathrm{~mm}$ to provide more space for the beam pipe and annular helium channel.

The expected level of magnetic fields in IR magnets suggests using a $\mathrm{Nb}_{3} \mathrm{Sn}$ superconductor. This superconductor has the most appropriate combination of the critical

\footnotetext{
${ }^{2}$ It should be noted that such values of beam-beam parameter were already achieved in $e^{+} e^{-}$machines.
}

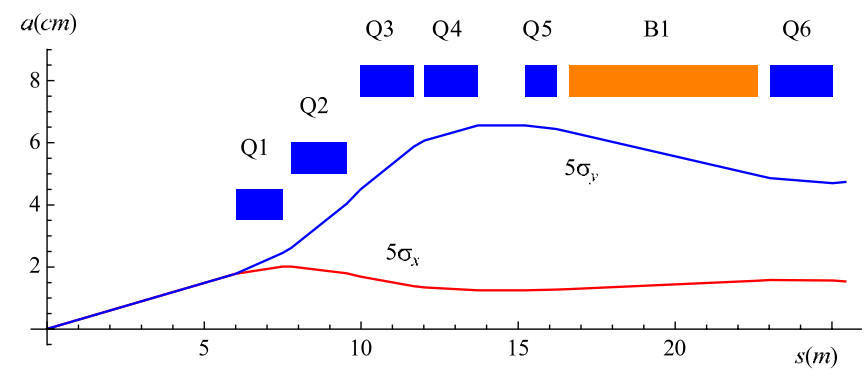

FIG. 3. Beam sizes and aperture of the FF magnets.

parameters including the critical current density $J_{c}$, the critical temperature $T_{c}$, and the upper critical magnetic field $B_{c 2}$ [12]. Cu-stabilized multifilament $\mathrm{Nb}_{3} \mathrm{Sn}$ strands with $J_{c}(12 \mathrm{~T}, 4.2 \mathrm{~K}) \sim 3000 \mathrm{~A} / \mathrm{mm}^{2}$, strand diameter $0.7-1.0 \mathrm{~mm}$, and the $\mathrm{Cu} / \mathrm{non}-\mathrm{Cu}$ ratio $\sim 0.9-1.1$ are commercially produced at the present time by industry in long length [13].

\section{A. IR QUADRUPOLES}

The IR doublets are made of relatively short quadrupoles (no more than $2 \mathrm{~m}$ long) to optimize their aperture according to the beam size variation and allow for placement of protecting tungsten masks between them. The first two quadrupoles in Fig. 3 are focusing ones and the next three are defocusing ones. The space between the 4th and 5 th quadrupoles is reserved for beam diagnostics and correctors.

The cross sections of MC IR quadrupoles based on twolayer shell-type $\mathrm{Nb}_{3} \mathrm{Sn}$ coils and cold iron yokes are shown in Fig. 4. Their parameters are summarized in Table II. All the designs use $16.3 \mathrm{~mm}$ wide cable made of 37 strands $0.8 \mathrm{~mm}$ in diameter. Strand $J_{c}(12 \mathrm{~T}, 4.2 \mathrm{~K})$ after cabling is $2750 \mathrm{~A} / \mathrm{mm}^{2}$ and the $\mathrm{Cu} /$ non-Cu ratio is 1.17 [14]. To maximize the iron contribution to the quadrupole field gradient, it is separated from the coils by thin $10 \mathrm{~mm}$ spacers. The two-layer coil design and the total coil width were selected based on the results of $\mathrm{Nb}_{3} \mathrm{Sn}$ cable and coil $\mathrm{R} \& \mathrm{D}$.

The nominal field in magnet coils is $\sim 11-12 \mathrm{~T}$, whereas the maximum field is reaching $\sim 13-15 \mathrm{~T}$. As can be seen, all magnets have $\sim 12 \%$ margin at $4.5 \mathrm{~K}$, which is sufficient (a)

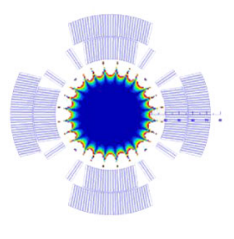

(b)

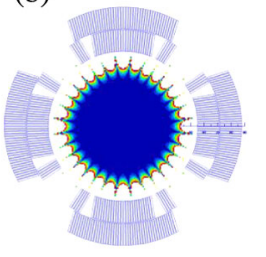

(c)

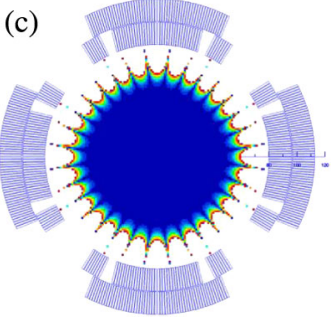

FIG. 4. Cross sections and a good-field region of Q1 (a), Q2 (b), and Q3-Q5 (c) quadrupoles. The dark blue color corresponds to the field error $|\delta B / B|<10^{-4}$. 
TABLE II. IR quadrupole parameters.

\begin{tabular}{llcrr}
\hline \hline Parameter & Unit & Q1 & Q2 & Q3 \\
\hline Coil aperture & $\mathrm{mm}$ & 80 & 110 & 160 \\
Nominal gradient & $\mathrm{T} / \mathrm{m}$ & 250 & 187 & -130 \\
Nominal current & $\mathrm{kA}$ & 16.61 & 15.3 & 14.2 \\
Quench gradient @ 4.5 K & $\mathrm{T} / \mathrm{m}$ & 281.5 & 209.0 & 146.0 \\
Quench gradient @ $1.9 \mathrm{~K}$ & $\mathrm{~T} / \mathrm{m}$ & 307.6 & 228.4 & 159.5 \\
Coil quench field @ $4.5 \mathrm{~K}$ & $\mathrm{~T}$ & 12.8 & 13.2 & 13.4 \\
Coil quench field @ $1.9 \mathrm{~K}$ & $\mathrm{~T}$ & 14.0 & 14.4 & 14.8 \\
Magnetic length & $\mathrm{m}$ & 1.5 & 1.7 & 1.7 \\
\hline \hline
\end{tabular}

for the stable operation with the average heat deposition in the magnet midplanes up to $1.7 \mathrm{~mW} / \mathrm{g}$. Operation at $1.9 \mathrm{~K}$ would increase the magnet margin to $\sim 22 \%$ and their quench limit by a factor of 4 .

The quench gradient and, respectively, operation margin of the IR quadrupoles at $4.5 \mathrm{~K}$ can be slightly increased if necessary by using wider (for example, three or four layers) and thus more complicated coils.

Geometrical field harmonics for IR quadrupoles Q1-Q5 are presented in Table III.

The accelerator field quality is achieved within the circles (blue areas in Fig. 4) equal to $2 / 3$ of the corresponding coil aperture. Saturation of the iron yoke and magnetization of cable and coil components and coil support structure will contribute to $b_{6}$. However, due to the fact that these magnets will operate at a constant field gradient, all these components can be easily compensated by appropriately tuning the quadrupole coil geometry.

The designs and parameters (mainly high operating field and large operating margin) of the MC FF quadrupoles are quite challenging and thus need to be practically demonstrated. Since they are close to the parameters of quadrupoles being developed by US-LARP collaboration for the LHC luminosity upgrade $[15,16]$, the results of LARP magnet R\&D will be applicable to the MC IR quadrupoles.

\section{B. IR dipoles}

The vertical elongation of the beam makes requirements to the IR dipoles quite different from those to the arc dipoles where the horizontal aperture must be larger due to the orbit sagitta and large dispersion contribution to the beam size. This allows using the traditional large-aperture $\cos \theta$ design with a sufficiently thick inner tungsten liner to protect the cold mass from the muon decay products.

TABLE III. Geometrical harmonics at $R_{\text {ref }}\left(10^{-4}\right)$.

\begin{tabular}{lrcc}
\hline \hline Harmonic \# & $\mathrm{Q} 1$ & $\mathrm{Q} 2$ & $\mathrm{Q} 3$ \\
\hline$R_{\text {ref }}(\mathrm{mm})$ & 27 & 37 & 53 \\
$b_{6}$ & 0.000 & 0.000 & 0.000 \\
$b_{10}$ & -0.034 & 0.002 & 0.002 \\
$b_{14}$ & 0.862 & 0.090 & 0.086 \\
\hline \hline
\end{tabular}

An alternative approach is the open midplane design concept, proposed for the MC storage ring dipoles [17], which allows the decay electrons to pass between the superconducting coils and be absorbed in high- $Z$ rods cooled at liquid nitrogen or possibly at room temperatures and placed far from the coils. This reduces heat deposition in the coils and-potentially-background fluxes in the central tracker of the detector.

To remove $95 \%$ of the radiation, the full gap between the poles should be at least $5 \sigma_{y}$ or $6 \mathrm{~cm}$. This large gap limits the bending field which can be achieved with $\mathrm{Nb}_{3} \mathrm{Sn}$ coils and make it more difficult to attain an acceptable field quality in the required aperture.

Several options were considered for an open midplane dipole based on a $\mathrm{Nb}_{3} \mathrm{Sn}$ superconductor with the required bending field of $8 \mathrm{~T}$, good field quality in the aperture with $100 \mathrm{~mm}$ in vertical direction and $50 \mathrm{~mm}$ in horizontal direction, and appropriate margin at $4.5 \mathrm{~K}$. The cross sections of two-layer $\cos \theta$ dipole design and most viable four-layer open-midplane dipole design are shown in Fig. 5. The main parameters of $\cos \theta$ and open-midplane dipoles are reported in Table IV. Both dipole designs are based on $14.7 \mathrm{~mm}$ wide cable with 28 strands $1.0 \mathrm{~mm}$ in diameter [14]. Strand $J_{c}(12 \mathrm{~T}, 4.2 \mathrm{~K})=2750 \mathrm{~A} / \mathrm{mm}^{2}$ includes possible $\sim 10 \%$ cabling degradation and the $\mathrm{Cu} /$ non-Cu ratio is 1.0 .

Geometrical field harmonics at the corresponding reference radii for IR dipoles $\mathrm{B} 1$ based on two alternative magnet designs are presented in Table V. In the traditional $\cos \theta$ design, the good field quality is provided within the circle with a radius of $60 \mathrm{~mm}$ [blue area in Fig. 5(a)]. In the open-midplane design the accelerator field quality is provided within a required elliptical area with $50 \mathrm{~mm}$ horizontal and $110 \mathrm{~mm}$ vertical size [blue area in Fig. 5(b)]. In this design it was achieved by an appropriate combination of relatively large values of low-order geometrical harmonics. As in the case of IR quadrupoles, the saturation of iron yoke and the magnetization of cable and coil components and coil support structure will contribute to the low-order field harmonics, mainly to $b_{3}$ and $b_{5}$. All these contributions will be compensated by reoptimizing the low-order harmonics at the operating field.

As it follows from Table IV, the traditional $\cos \theta$ design provides larger maximum field and, respectively, larger
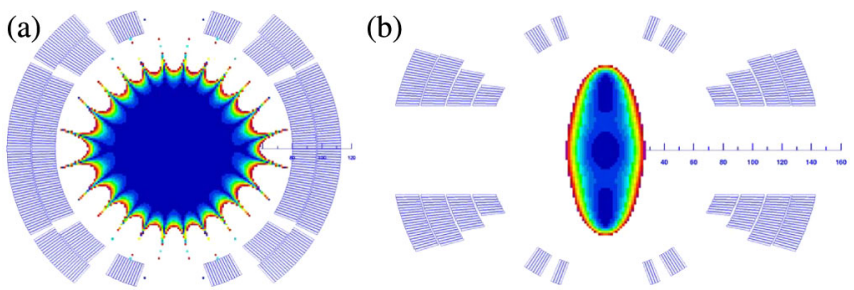

FIG. 5. Cross sections and a good-field region of the dipole B1 based on $\cos \theta$ (left) and open midplane (right) coil design. The dark blue color corresponds to the field error of $|\delta B / B|<10^{-4}$. 
TABLE IV. IR dipole parameters.

\begin{tabular}{llcc}
\hline \hline Parameter & Unit & $\cos \theta$ & Open midplane \\
\hline Coil aperture & $\mathrm{mm}$ & 160 & 160 \\
Gap & $\mathrm{mm}$ & 0 & 55 \\
Nominal field & $\mathrm{T}$ & 8 & 8 \\
Nominal current & $\mathrm{kA}$ & 8.28 & 17.85 \\
Quench field @ 4.5 K & $\mathrm{T}$ & 12.46 & 9.82 \\
Magnetic length & $\mathrm{m}$ & 6 & 6 \\
\hline \hline
\end{tabular}

TABLE V. Geometrical harmonics $\left(10^{-4}\right)$.

\begin{tabular}{lcc}
\hline \hline Harmonic \# & $\cos \theta$ & Open midplane \\
\hline$R_{\text {ref }}(\mathrm{mm})$ & 53 & 40 \\
$b_{3}$ & 0.04 & -5.88 \\
$b_{5}$ & 0.03 & -18.32 \\
$b_{7}$ & 0.40 & -17.11 \\
$b_{9}$ & 0.60 & -4.61 \\
\hline \hline
\end{tabular}

operation margin than the open midplane design. It is also more straightforward from the viewpoint of fabrication and cold mass cooling. However, the aperture of this magnet, the coil volume, and the Lorentz force level depend on the absorber size which make this design also quite challenging. Both designs require significant $\mathrm{R} \& \mathrm{D}$ efforts.

\section{ENERGY DEPOSITION IN MAGNETS}

Energy deposition and detector backgrounds are simulated with the MARS15 code [18]. All the related details of geometry, materials distributions, and magnetic fields are implemented into the model for lattice elements and tunnel in the \pm 200 - $m$ region from IP, detector components [19], experimental hall, and machine-detector interface. To protect SC magnets and detector, tungsten masks in the interconnect regions, liners in magnet apertures (wherever

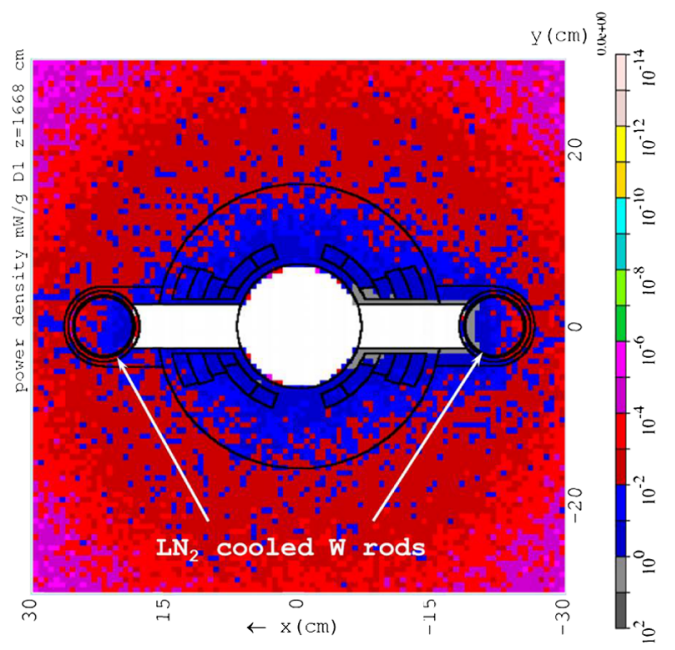

FIG. 7. Power density ( $\mathrm{mW} / \mathrm{g}$ ) in B1 dipole for case (iii).

needed), and a sophisticated tungsten cone inside the detector [5] were implemented into the model and carefully optimized. The muon beam with parameters cited in Table I was assumed to be aborted after 1500 turns when the luminosity is reduced by a factor of $\sim 6$.

Three cases were considered: (i) "standard" when $10-\mathrm{cm}$ long tungsten masks with $5 \sigma_{x, y}$ elliptic openings are put in the IR magnet interconnect regions; (ii) with additional tungsten liners inside the quadrupoles leaving a $5 \sigma_{x, y}$ elliptic aperture for the beam; (iii) as in the first case, but with the IR quadrupoles displaced horizontally by 0.1 of their apertures, so as to provide $\sim 2 \mathrm{~T}$ bending field. This additional field helps also facilitate chromaticity correction by increasing dispersion at the sextupoles, and deflect lowenergy charged particles from the detector.

Power density isocontours at shower maximum in the first quadrupole are shown in Fig. 6, while Fig. 7 displays such profiles in the IR dipole B1. Maximum values of power density in the most vulnerable magnets are
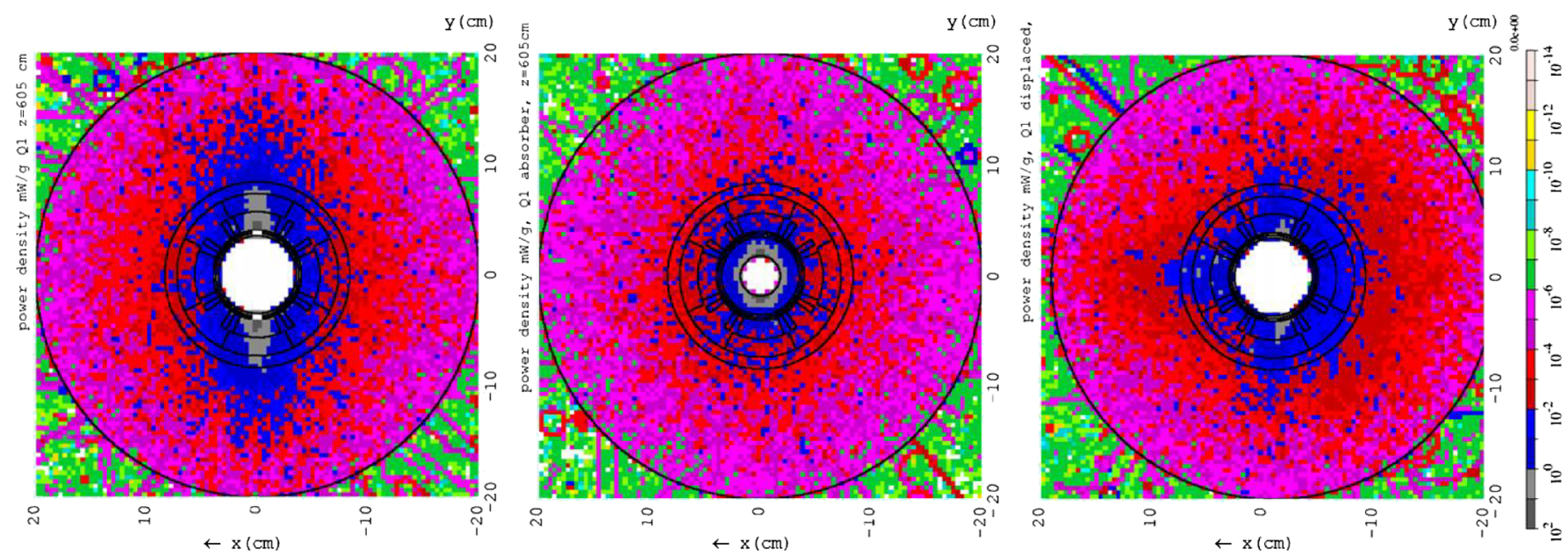

FIG. 6. Deposited power density in Q1 (mW/g) for three cases: standard (left), with absorbers inside (center), and with horizontal displacement (right). Larger radii are on the left of the plots. 
TABLE VI. Peak power density $(\mathrm{mW} / \mathrm{g})$ in most vulnerable magnets in three considered cases.

\begin{tabular}{llll}
\hline \hline Magnet & (i) & (ii) & (iii) \\
\hline Q1 & 5.0 & 1.0 & 3.0 \\
Q2 & 10. & 1.0 & 10. \\
Q5 & 3.7 & 2.0 & 3.7 \\
B1 & 3.0 & 2.6 & 1.9 \\
Q6 & 3.6 & 2.6 & 2.0 \\
\hline \hline
\end{tabular}

presented in Table VI. One can see that quadrupole displacement reduces power density but not enough to avoid using liners inside quadrupoles. Combining all three cases has a potential of keeping peak power density in the IR magnets below the quench limits of about $5 \mathrm{~mW} / \mathrm{g}$ with a necessary safety margin (typically a factor of 3 ).

\section{DETECTOR BACKGROUNDS}

Figure 8 compares calculated electron and gamma fluxes for the following cases: (left) no masks between magnets, $6^{\circ}$ cone with a $5 \sigma$ radius liner up to $2 \mathrm{~m}$ from IP; (center) $5 \sigma$ masks inserted between $\mathrm{FF}$ quads, cone angle increased to $10^{\circ}, 5 \sigma$ liner up to $1 \mathrm{~m}$ from IP; (right) same as above plus FF quad displacement.

The masks and increased cone angle reduce the electron and gamma fluxes by factors 300 and 20, respectively. Displacing the FF quads slightly increases the electron flux (by up to $50 \%$ ) but decreases the gamma flux by another factor of 15 , so the overall effect of quad displacement may be considered as positive.

Results of further optimization of the cone nose geometry are presented in Fig. 9. It shows gamma flux as a function of the angle of inner cone opening towards IP at the outer cone angle of $10^{\circ}$. For such a cone and a set of other optimal parameters as seen now, the maximum neutron fluence and absorbed dose in the innermost layer of the silicon tracker for a one-year operation are at a $10 \%$ level of that in the LHC detectors at the luminosity of $10^{34} \mathrm{~cm}^{-1} \mathrm{~s}^{-1}$. Photon fluence is several times higher than that at the LHC.
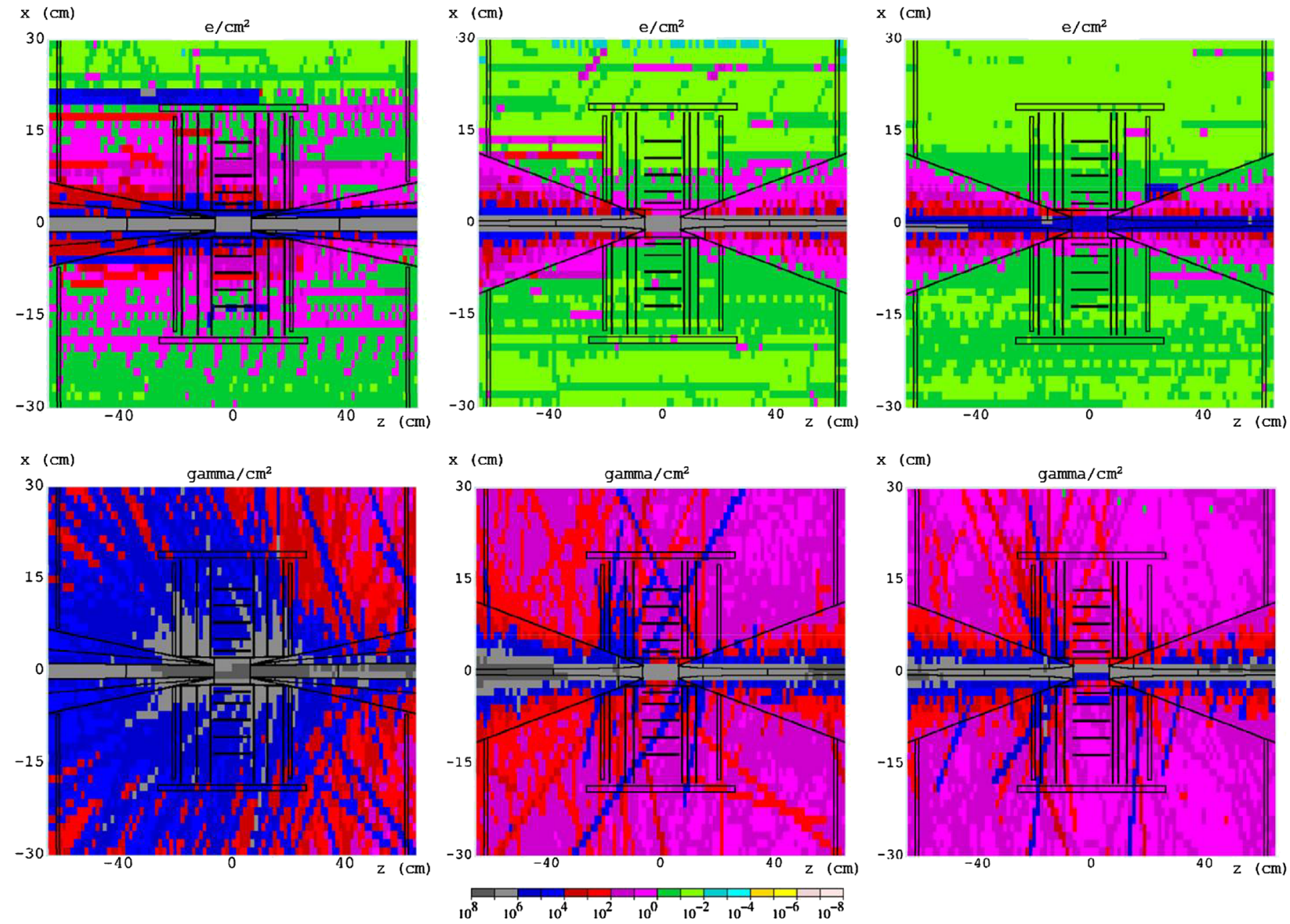

FIG. 8. Electron (top) and gamma (bottom) fluxes in the detector in three cases described in the text. 


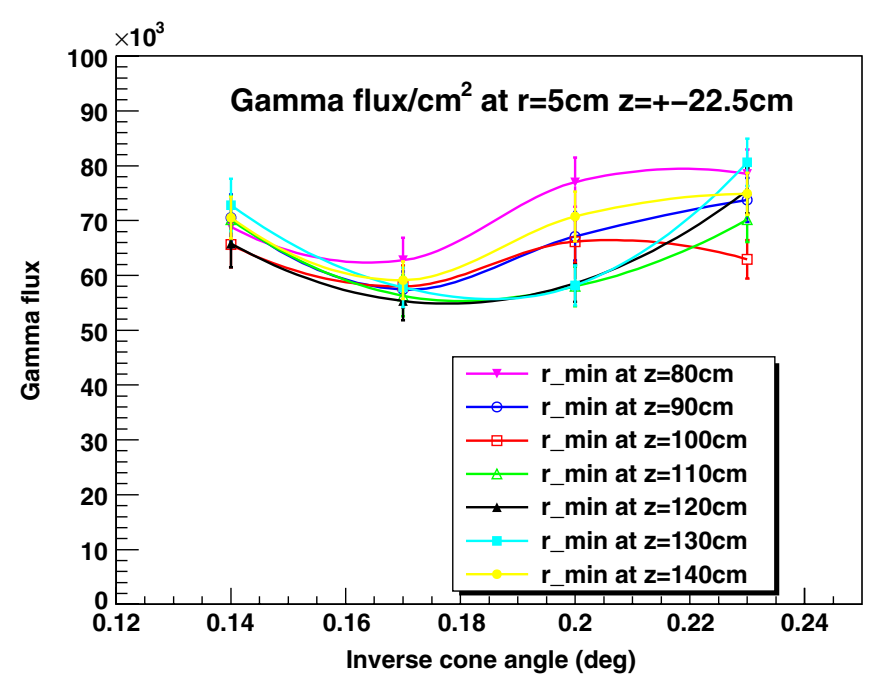

FIG. 9. Gamma flux vs inner cone angle at different positions of minimal aperture from IP.

\section{SUMMARY AND OUTLOOK}

The presented interaction region lattice is a part of the complete muon collider storage ring design which satisfies all requirements from the beam dynamics point of view in the considered case of $1.5 \mathrm{TeV}$ center-of-mass energy and the average luminosity of $10^{34} \mathrm{~cm}^{-2} \mathrm{~s}^{-1}$.

All the required IR magnets can be built using the $\mathrm{Nb}_{3} \mathrm{Sn}$ technology which is being developed for the LHC luminosity upgrade and shows very promising results for a muon collider storage ring and interaction regions. Using a combination of special measures (internal absorbers and masks), the heat deposition in IR magnets can be reduced below the quench limit of $\mathrm{Nb}_{3} \mathrm{Sn}$ magnets at $4.5 \mathrm{~K}$ with a safety margin.

With the proposed protective measures implemented in the machine-detector interface, the calculated backgrounds are comparable to those expected at LHC for the same luminosity.

Further studies and optimization of the $1.5 \mathrm{TeV}$ muon collider design need to be focused on: (i) feasibility studies and modeling of the open-midplane dipole design [the studies and development of large-aperture traditional dipole magnets with comparable operating parameters are supported by some other R\&D programs (see, for example, [20])]; (ii) reduction of detector backgrounds by optimizing parameters of the protective cone and other machinedetector interface elements; (iii) adding a collimation scheme to the muon collider lattice design, which actually should be extraction of the beam halo [21].

At the same time the work on a more challenging muon collider design with a 3-TeV center-of-mass energy has also been started. It will require even stronger SC magnets and will have to address such issues as the neutrinoinduced radiation [22].

\section{ACKNOWLEDGMENTS}

The authors are grateful to S. Geer, R. Palmer, and A. Tollestrup for many useful remarks and to Dr. K. Oide for kindly providing the detail of his MC design. This work was supported by Fermi Research Alliance, LLC under U.S. DOE Contract No. DE-AC02-07CH11359.

[1] G. I. Budker, in Proceedings of the VII International Conference on High Energy Accelerators, Yerevan, 1969 (Academy of Sciences of Armenia, Yerevan, 1970), Vol. I, pp. 33-39.

[2] C. Ankenbrandt et al., Phys. Rev. ST Accel. Beams 2, 081001 (1999).

[3] R\&D Proposal for National Muon Accelerator Program, Report No. FERMILAB-TM-2459-APC, 2010.

[4] S. Geer, Annu. Rev. Nucl. Part. Sci. 59, 347 (2009).

[5] C. J. Johnstone and N. V. Mokhov, in Proceedings at Snowmass 1996 (BNL-52503, Fermilab-Conf-96/092, LBNL-38946, 1996), pp. 226-229; in Proceedings of the Particle Accelerator Conference, Vancouver, BC, Canada, 1997 (IEEE, New York, 1997), pp. 414-416.

[6] J. Irwin et al., in Proceedings of the 1991 Particle Accelerator Conference, San Francisco, CA, 1991 (IEEE, New York, 1991), p. 2058.

[7] K. Oide (unpublished); https://mctf.fnal.gov/databases/ lattice-repository/collider-ring/oide.

[8] B. W. Montague, LEP Note No. 165, 1979.

[9] T. Sen and M. Syphers, SSCL Report No. 411, 1993.

[10] Y. Alexahin, E. Gianfelice-Wendt, and A. Netepenko, in Proceedings of the First International Particle Accelerator Conference, Kyoto, Japan (2010), TUPEB021.

[11] T. Sen et al., Report No. LARP-DOC-103, 2005.

[12] L. T. Summers et al., IEEE Trans. Magn. 27, 2041 (1991).

[13] J. A. Parrell et al., IEEE Trans. Appl. Supercond. 19, 2573 (2009).

[14] A. V. Zlobin et al., in Proceedings of the First International Particle Accelerator Conference, Kyoto, Japan (2010), MOPEB053.

[15] A. V. Zlobin et al., in Proceedings of the 11th European Particle Accelerator Conference, Genoa, 2008 (EPS-AG, Genoa, Italy, 2008).

[16] P. Wanderer, IEEE Trans. Appl. Supercond. 19, 1208 (2009).

[17] B. Parker et al., in Proceedings of the Particle Accelerator Conference, Chicago, IL, 2001 (IEEE, New York, 2001).

[18] N. V. Mokhov, http://www-ap.fnal.gov/MARS/.

[19] http://www.4thconcept.org/4LoI.pdf.

[20] G. de Rijk, in Proceedings of the 11th European Particle Accelerator Conference, Genoa, 2008 (Ref. [15]), pp. 2419-2421.

[21] A. I. Drozhdin et al., AIP Conf. Proc. 441, 242 (1998).

[22] N. V. Mokhov and A. Van Ginneken, J. Nucl. Sci. Technol. 1, 172 (2000). 